\title{
Erratum to: Monsters and I: The Case of Mixed Quotation
}

\author{
Kasia M. Jaszczolt and Minyao Huang
}

\section{Erratum to:}

Chapter 13 in: P. Saka, M. Johnson (eds.), The Semantics and Pragmatics of Quotation, Perspectives in Pragmatics, Philosophy \& Psychology 15, https://doi.org/10.1007/978-3-319-68747-6_13

The following material was inadvertently omitted from the beginning of footnote 27 , p. 380: Research leading to this paper was supported by the Leverhulme Trust Grant Expressing the Self: Cultural Diversity and Cognitive Universals (Grant ID/Ref: RPG-2014-017)

http://www.mml.cam.ac.uk/expressing-the-self.

The updated online version of this chapter can be found at https://doi.org/10.1007/978-3-319-68747-6_13 